\title{
APPLICATION OF THE CLOSEST POINT PROJECTION METHOD TO SOLUTION OF REYNOLD'S LUBRICATION EQUATIONS ON EVOLVING SURFACES
}

\author{
E. ZIPUNOVA, A. IVANOV, E. SAVENKOV* \\ Keldysh Institute of Applied Mathematics, Russian Academy of Sciences, Moscow, Russia \\ *Corresponding author. E-mail: e.savenkov@googlemail.com
}

DOI: $10.20948 /$ mathmontis-2020-47-9

Summary. The paper considers a computational algorithm for solution of surface PDEs defined on the evolving surfaces. The basis of the algorithm is a finite element version of the closest point projection method. The method is applied to the Reynold's lubrication equation which governs fluid flow in thin fractures. The closest point approach is used for description of fracture mid-surface evolution and for construction of the embedding equation. We describe algorithmic details of the proposed approach as well as a number of numerical experiments which demonstrates robustness of the method.

\section{INTRODUCTION}

Currently, hydraulic fracturing (HF) is one of the most widely used methods of oil and gas reservoir stimulation. The essence of the technology is an injection of special fluid into reservoir in order to create an artificial fracture of considerable area (length $\sim 100 \mathrm{~m}$, height $\sim 10 \mathrm{~m}$, average opening $\sim 5-10 \mathrm{~mm}$ ). The fracture is filled with a proppant - calibrated artificial or natural sand-like granular material. The result of HF procedure is an artificial flow channel connected to the production or injection well with large inflow area and high permeability. This provides a significant increase in inflow of reservoir fluid to the well. Engineering aspects of technology are considered, for example, in [1].

Mathematical description of the hydraulic fracture evolution during its development comes down to solution of complex coupled problem which includes (among other groups of equations) flow equations of (usually non-Newtonian) for fluid in the evolving fracture.

A number of models for HF evolution are known. Most general of them (see, e.g., [2]) assume that:

- fracture mid-surface is an arbitrary sufficiently smooth surface with boundary;

- at a fixed point of the mid-surface its opening is defined by reaction of the surrounding medium and pressure in the fracture;

- during the hydraulic fracturing procedure, the fracture evolves, and the exact way of this evolution is not known in advance (in other words, the fracture mid-surface is a part of the solution of complete problem);

- the fracture mid-surface is not flat - fracture can switch direction of propagation locally; the direction of its propagation may be different at different points on its front.

Note that common approaches to solve the problem of fracture propagation in an elastic medium are based on boundary integral equations. In this case it turns possible to solve both 
fracture flow equations and elasticity equations in the surrounding medium using single surface computational mesh defined on the fracture mid-surface. This simplifies overall algorithm for the complete problem. In the case when the medium is, for example, heterogeneous, these methods cannot be applied - and the mesh-based methods like, e.g. finite element method are more preferable. In that case an additional computational mesh has to be introduced for solution of surface PDE. The same situation arises when realistic fluid flow models in reservoir has to be considered, see, e.g. [3].

Another approach is to use the so called embedding methods when surface PDE is solved using the same spatial mesh as a problem in the surrounding medium. A number of such methods are known. In the works $[4,5,6,7,8]$ an original method was proposed for solving surface PDEs based on surface representation using closet point projection operator. The essence of the method is that closest point projection operator is used to extend surface PDE into the surrounding space to obtain the so called embedding PDE. Further, this embedding PDE is approximated by a suitable difference method on a mesh which, generally, is not consistent with surface geometry. As a solution to the original problem on the surface, the trace of $3 \mathrm{D}$ embedding equation is considered. At the same time, the closet point projection operator is used to approximate Dirichlet (or Neumann) boundary conditions defined on the boundary of both the original surface and the three-dimensional domain, where embedding PDE is solved.

In this paper, we consider the finite element version of the closet point projection method to solve Reynold's lubrication equations which governs fluid flow inside evolving fracture.

In contrast to the works cited above, the finite element method is used a basic approximation scheme. More detailed description of the algorithm as well as numerical examples are presented in $[19,20]$.

\section{CLOSEST POINT PROJECTION METHOD}

This section briefly describes the main ideas of the closest point projection method for numerical solution of surface PDEs. The method was proposed and developed in $[4,5,8,6,7]$. It uses implicit representation of the surface and is based on the extension of surface PDE into the space. To construct such extension the closest point projection operator is used rather then commonly used level set method.

In this section, for simplicity, we consider model boundary value problem for a parabolic equation with Laplace-Beltrami operator (see, for example, [21]) defined on a curved surface F with boundary:

$$
\frac{\partial u}{\partial t}-\Delta_{\mathrm{F}} u=0
$$

complemented by the initial and boundary condition of the desired type.

We assume that the surface $F$ is entirely located inside the spatial domain $\Omega \subset \mathrm{R}^{3}$. Suppose that for an arbitrary point $\mathbf{x} \in \Omega$, a point $\mathbf{x}_{\mathrm{cp}}$ is the nearest to $x$ point on the surface F ,

$$
\mathbf{x}_{\mathrm{cp}}=\operatorname{argmin}_{\mathbf{y} \in \mathrm{F}} \mathrm{P} \mathbf{y}-\mathbf{x} \mathrm{P}
$$


where $\mathrm{P} \cdot \mathrm{P}$ is the Euclidean norm in $\mathrm{R}^{3}$. The point $\mathbf{x}_{\mathrm{cp}}$ is called the closest point projection of the point $\mathbf{x}$ onto the surface $\mathrm{F}$, and the corresponding operator will be denoted by $\mathrm{P}$,

$$
\mathbf{x}_{\mathrm{cp}}=\mathrm{P} \mathbf{x}
$$

The operator $\mathrm{P}$ is vector-valued: it maps spatial domain $\Omega$ to the surface $\mathrm{F}$, considered as a subset of $\Omega \subset \mathrm{R}^{3}$.

If the sign distance function $d_{\mathrm{F}}(\mathbf{x})$ can be specified for the surface $\mathrm{F}$ (for example, if $\mathrm{F}$ is oriented surface without boundary), then the following representaion for the operator $\mathrm{P}$ is valid:

$$
\mathrm{P}(\mathbf{x})=\mathbf{x}-d_{\mathrm{F}}(\mathbf{x}) \nabla d_{\mathrm{F}}(\mathbf{x}), \quad d(\mathbf{x})=\mathrm{P} \mathbf{x}-\mathrm{Px} \mathrm{P} .
$$

Just like the sign distance function (or a pair of such functions in the case of a surface with boundary), the projector $\mathrm{P}$ uniquely defines surface $\mathrm{F}$,

$$
\mathrm{F}=\{\mathbf{x} \in \Omega: \quad \mathbf{x}=\mathrm{Px}\} .
$$

However, the closest point projection approach is more general: it allows one to describe geometry of surface with boundary, non-orientable manifolds or manifolds of codimension greater than one (i.e. curves and points which are codimension 2 and 3 objects in $3 \mathrm{~d}$ case - as well as union of objects of different codimension).

Using projector $\mathrm{P}$, it is easy to construct an extension of an arbitrary function defined on the surface to the spatial domain $\Omega$ : for an arbitrary function $u$ defined on a surface, its continuation $\mathrm{E}[u]$ in $\Omega$ is defined as

$$
\mathrm{E}[u](\mathbf{x})=u(\mathrm{P} \mathbf{x}), \quad \mathbf{x} \in \Omega .
$$

In both cases, the operator $\mathrm{E}$ is a projector in the sense that $\mathrm{E}^{2}=I$, where $I$ is the identity operator. Note that:

- for an arbitrary function in $\Omega$ which is constant along the direction normal to $F$,

$$
\left.(\nabla u)\right|_{\mathrm{F}}=\nabla_{\mathrm{F}}\left(\left.u\right|_{\mathrm{F}}\right)
$$

- for an arbitrary vector field in $\Omega$ tangent to the surface $F$,

$$
\left.(\nabla \cdot \mathbf{q})\right|_{\mathrm{F}}=\nabla_{\mathrm{F}} \cdot\left(\left.q\right|_{\mathrm{F}}\right) \text {. }
$$

Then, due to the properties of the projector $\mathrm{P}$ and the extension operator $\mathrm{E}$ :

$$
\nabla \mathrm{E}[u](\mathbf{x})=\nabla u(\mathrm{Px})=\nabla_{\mathrm{F}} u .
$$

Since the $\mathrm{E}[u](\mathbf{x})$ is constant along directions normal to the surface, the vector field $\nabla \mathrm{E}[u](\mathbf{x})$ is tangent to $\mathrm{F}$. Hence,

$$
\nabla \cdot[\nabla \mathrm{E}[u](\mathbf{x})]=\nabla \cdot[\nabla u(\mathrm{Px})]=\nabla_{\mathrm{F}} \cdot \nabla_{\mathrm{F}} u .
$$

Similar extensions can be constructed for more complex elliptic operators defined in the surface, see [9]. 
Thus, the original equation (1) can be extended to the spatial domain $\Omega$ to define the so called embedding equation. Further, the embedding equation is approximated by a suitable difference method on a three-dimensional mesh introduced in $\Omega$ and, generally, inconsistent with the surface geometry. Solution of the original problem on the surface can restored as a trace of the solution of 3D problem on the surface. A rigorous justification for the constructions described above is presented in [9]. Details of the method are given in the papers cited above.

Following the described approach the embedding equation which corresponds to (1) reads:

$$
\frac{\partial u}{\partial t}+\nabla \cdot(\nabla \mathrm{E}[u])=\mathrm{E}[f], \quad \mathbf{x} \in \Omega_{\mathrm{F}} .
$$

The trace of the solution of this equation on $\mathrm{F}$ is the solution of the equation (1).

Regarding domain $\Omega$, it is assumed that (i) it is small in the sense that the closest point projection of an arbitrary point from $\Omega$ is well defined and (ii) the domain $\Omega$ includes the surface $F, \Omega \supset F$, and the distances from the boundary points of $\Omega$ to surface are positive. In other words, all points on the surface are interior points of the domain.

If the surface $\mathrm{F}$ is a surface without boundary, the value of the solution $u(t, \mathbf{x})$ of the embedding PDE (2) at the points lying on the surface $\mathrm{F}$ will coincide with the solution of the original surface PDE (1) (in this case $\partial \mathrm{F}=\varnothing$ and the problem (1) is the Cauchy problem). Otherwise it is required to provide embedding PDE (2) with boundary conditions defined in a suitable way. This can be done in different ways. In [8] a convenient way to adress this issue in the discrete setting. In the context of the finite element method the corresponding questions were adressed in [10].

\section{PROBLEM STATEMENT}

This section presents the mathematical formulation of the problem. In subsequent section with consider a flow model itself and geometrical model for fracture mid-surface evolution.

\subsection{Flow model in fracture}

Consider a one-sided surface $\overline{\mathrm{F}}$ with boundary $\partial \mathrm{F}$ immersed into three-dimensional space $\mathrm{R}^{3}$. Let $\overline{\mathrm{F}}=\mathrm{F} \cup \partial \mathrm{F}$, where an open domain $\mathrm{F}$ is an internal part of the surface, $\partial \mathrm{F}$ - its boundary. In some cases, we will identify the notation $\mathrm{F}$ and $\overline{\mathrm{F}}$. We assume that $\mathrm{F}$ and its boundary have the required smoothness.

The surface $\overline{\mathrm{F}}$ is assumed to be immersed into spatial domain $\Omega$. Let for each point $\mathbf{x} \in \Omega$ a closest point projector $\mathrm{P}$ onto $\overline{\mathrm{F}}$ is uniquelly defined (see section 2). Depending on the location of the point, its projection onto $\overline{\mathrm{F}}$ belongs to either $\mathrm{F}$ or $\partial \mathrm{F}$. This allows us to represent $\Omega$ as a union $\Omega=\Omega_{\mathrm{F}} \cup \Omega_{\partial \mathrm{F}}$, where

$$
\Omega_{\mathrm{F}}=\{\mathbf{x} \in \Omega: \mathrm{Px} \in \mathrm{F}\}, \quad \Omega_{\partial \mathrm{F}}=\{\mathbf{x} \in \Omega: \mathrm{P} \mathbf{x} \in \partial \mathrm{F}\} .
$$

The boundary $\partial \Omega_{\mathrm{F}}$ of the $\Omega_{\mathrm{F}}$ can be represented as

$$
\partial \Omega_{\mathrm{F}}=\Gamma_{\mathrm{F}} \cup \Gamma_{\partial \mathrm{F}}, \quad \Gamma_{\mathrm{F}}=\partial \Omega_{\mathrm{F}} \cap \partial \Omega, \quad \Gamma_{\partial \mathrm{F}}=\partial \Omega_{\mathrm{F}} \backslash \Gamma_{\mathrm{F}} .
$$


In the case of evolving fracture geometry, the constructions above are generally the same, except that the computational domain now depends on time. That is, the problem is solved in the domain $\mathrm{F}=\mathrm{F}_{t} \equiv \mathrm{F}(t)$, where $t$ is the time, $\Omega=\Omega_{t} \equiv \Omega(t), \Omega_{\mathrm{F}}=\Omega_{\mathrm{F}, t} \equiv \Omega_{\mathrm{F}}(t)$, etc. Additionally, we assume that for all moments of time, all previously considered time-dependent domains, as well as the mid-surface of the fracture are located inside some spatial and time-independent domain $\tilde{\Omega}$. Let us note that the dependency of the mid-surface $\mathrm{F}$ on time cannot to be an arbitrary. Corresponding clarifications will be given below.

The Reynold's lubrication equation of fluid flow in fracture has the form:

$$
\frac{\partial \rho w}{\partial t}+\operatorname{div}\left(-\frac{1}{12 v} \rho w^{3} \nabla p\right)=f_{\mathrm{m}}, \quad \mathbf{x} \in \mathrm{F}_{t},
$$

where $\rho$ is the fluid density; $w=w(\mathbf{x})-$ fracture opening, $\mathbf{x} \in \mathrm{F}-$ point of the fracture mid-surface $\mathrm{F} ; v$ - fluid viscosity, $p=p(\mathbf{x})$ - its pressure, $f_{\mathrm{m}}$ - mass rate of external sources.

We assume that the fluid density linearly depends on pressure, i.e.

$$
\rho=\rho_{0}\left[1+c_{\mathrm{f}}\left(p-p_{0}\right)\right]
$$

where $\rho_{0}$ and $p_{0}$ are reference values of density and pressure, $c_{\mathrm{f}}$ is compressibility of the fluid.

The equation (3) is supplemented with initial and boundary condition of the form

$$
p(\mathbf{x}, t=0)=p_{\text {ini }}(\mathbf{x}),\left.\quad p\right|_{\partial \mathrm{F}}=p_{\partial \mathrm{F}} .
$$

Further, we assume that the opening $w$ is always positive,

$$
w=w(\mathbf{x}) \ldots w_{0}>0, \quad \mathbf{x} \in \mathrm{F} \cup \partial \mathrm{F} .
$$

In the simplest case we can assume that fracture opening $w$ does not depend on pressure. In a more realistic setting, opening is a function of pressure. This relationship reflects the fact that the fracture is located inside an elastic medium that deforms when the pressure in the fracture changes. In this paper it is assumed that this dependency is linear, i.e.

$$
w(\mathbf{x})=\mathrm{W}(\mathbf{x} ; p(\mathbf{x})), \quad \mathrm{W}(\mathbf{x} ; p)=w_{\text {ref }}(\mathbf{x})\left(1+c_{\mathrm{w}}\left[p(\mathbf{x})-p_{\text {ref }}\right]\right),
$$

where $w_{\text {ref }}(\mathbf{x})$ is fracture opening at reference pressure $p_{\text {ref }}, c_{\mathrm{w}}$ is coefficient describing "compressibility" of fracture (more precisely, the medium containing the fracture).

\subsection{Model for surface evolution}

Evolution of the surface $\mathrm{F}_{t}$ in the formulation (3) can not be an arbitrary. This section presents relevant assumptions, both of the fundamental and technical character. They reflect the specifics of the problem under consideration, namely, the fact that $\mathrm{F}_{t}$ is the mid-surface of an evolving fracture.

Let the problem (3) be solved on the time interval $t \in[0, T]$ and for $t_{1} . . t_{2}$ condition $\mathrm{F}_{t_{1}} \subset \mathrm{F}_{t_{2}}$ holds. In other words, a family of surfaces corresponding to smaller times, is 
contained inside a surface corresponding to any larger time. Or, which is the same, the surface may evolve only due to the movement of its boundary.

We assume that:

- at any time $t \in[0, T]$ the surface $\mathrm{F}_{t}$ is entirely located inside some spatial time-independent domain $\Omega$;

- the evolution of the surface F is "smooth", i.e., at any time the surface can be smoothly and one-to-one mapped onto, for example, a unit disk in $\mathrm{R}^{2}$. In particular, during its evolution $\mathrm{F}$ should not have self-intersections, and so on.

In this case, the family of surfaces $\left\{\mathrm{F}_{t}, t . .0\right\}$ can be represented as a union of the "initial" surface $F \equiv F_{0}$ and the "trace" of the movement of the surface boundary, i.e.

$$
\mathrm{F}_{t}=\left\{\mathrm{F}_{0} \cup \gamma\left(t^{\prime}\right) \mid 0, t^{\prime}, t\right\},
$$

where $\mathrm{F} \equiv \mathrm{F}_{0}$ is the surface at $t=0, \gamma(t) \equiv \partial \mathrm{F}_{t}$.

Thus, the evolution of the surface is determined by the motion of its boundary $\gamma(t)$. In the following we assume that at every moment of time, on the curve $\gamma(t)$ the velocity vector field $\mathbf{v}=\mathbf{v}(\mathbf{x}, t), \quad \mathbf{x} \in \gamma(t)$ is defined which governs fracture evolution. Movement of the (Lagrangian) point of the boundary is described by the equation

$$
\frac{d \mathbf{x}}{d t}=\mathbf{v}(\mathbf{x}, t),\left.\quad \mathbf{x}\right|_{t=0}=\mathbf{x}_{0} \in \gamma(0) .
$$

We assume that $\mathbf{v}=\mathbf{v}(\mathbf{x}, t)$ (i) is smooth function of point $\mathbf{x} \in \gamma(t)$ for $t \in[0, T]$ and (ii) is a smooth function of time for each fixed (Lagrangian) point on the boundary.

Such a model of surface evolution corresponds to the problem of fracture dynamics. In this case the direction of surface evolution is known only at the points of its boundary (fracture front) and is determined by the appropriate fracture criteria (see e.g. [11, 12]).

Note that in a number of cases (in particular, for the purposes of theoretical analysis) it is convenient to assume that the velocity field $\mathbf{v}(\mathbf{x}, t), \mathbf{x} \in \partial \mathrm{F}_{t}$ is a trace on $\partial \mathrm{F}_{t}$ of some smooth vector field $\mathbf{V}=\mathbf{V}(\mathbf{x}, t)$ without singular points which is defined in the domain $\Omega$ containing the family of surfaces $\mathrm{F}_{t}$ at all instants of time $t \in[0, T]$. The natural requirement for the field $\mathbf{V}=\mathbf{V}(\mathbf{x}, t)$ is that that it is tangent to surfaces $\mathrm{F}_{\tilde{t}}$ for all $\tilde{t}<t$ (in other words, it does not change already formed fracture mid-surface). Under a suitable choice, the field $\mathbf{V}$ generates a smooth and one-to-one mapping of the surface $\mathrm{F} \equiv \mathrm{F}_{0}$ onto the surface $\mathrm{F}_{t}$ for any $t \in(0, T]$.

\section{COMPUTATIONAL ALGORITHM}

\subsection{Formal time approximations}

We write the problem (3) in the operator form as 


$$
\begin{gathered}
\frac{\partial \varphi(u)}{\partial t}+\mathrm{A}(w(u) ; u)=f, \quad \mathbf{x} \in \mathrm{F}_{t}, \\
\left.u\right|_{\partial \mathrm{F}}=g, \quad \mathbf{x} \in \partial \mathrm{F}_{t},
\end{gathered}
$$

with initial condition

$$
u(\mathbf{x}, t=0)=u_{0}(\mathbf{x}), \quad \mathbf{x} \in \mathrm{F}_{0} .
$$

Let the problem (6a) be solved for $t \in[0, T]$. We divide interval $[0, T]$ it into intervals (time steps) $\Delta t$, so that

$$
0=t_{0}<\Delta t<\ldots<n \Delta t=t_{n}<\ldots N \Delta t=t_{N}=T .
$$

According to section 3.2 at time point $t$ the solution $u_{n}=u\left(t_{n}\right)$ is defined in the domain $\mathrm{F}_{t_{n}} \equiv \mathrm{F}_{n}$, moreover, $\mathrm{F}_{n} \subset \mathrm{F}_{n+1}$.

Semi-discrete (in time) approximation of the problem (6a) on the interval $\left[t_{n}, t_{n+1}\right]$ can be defined as

$$
\frac{\varphi\left(u_{n+1}\right)-\mathrm{E}\left[\varphi\left(u_{n}\right)\right]}{\Delta t}+\mathrm{A}\left(w\left(u_{n+1}\right) ; u_{n+1}\right)=f_{n+1}, \quad \mathbf{x} \in \mathrm{F}_{n+1} .
$$

Here $\mathrm{E}[\cdot]$ is the continuation operator that maps functions, defined in $\mathrm{F}_{n}$ to functions defined in $\mathrm{F}_{n+1}$. For the problem to be well-posed, the continuation operator must satisfy certain smoothness and boundedness properties. In terms of Sobolev spaces, such properties of the continuation operator are formulated in [13].

In this paper, a rigorous theoretical justification of the proposed method used is not given, but it can be expected that the conditions necessary for its correctness are satisfied since:

- for smooth domains with smooth boundary natural smoothness properties of the solution coincide with those ones for the case of planar domains (subsets of $\mathrm{R}^{2}$ ). Moreover, the surface $\mathrm{F}_{t}$ for all $t$ can be mapped smoothly to the two-dimensional domain $\tilde{\mathrm{F}_{t}} \subset \mathrm{R}^{2}$ (and even to a time-independent canonical domain, for example, a disk of a given radius)

- the vector field $\mathbf{V}$ that governs mid-surface evolution (see section 3.2) defines a smooth mapping $\mathrm{F} \equiv \mathrm{F}_{0}$ onto $\mathrm{F}_{t}$.

We also note the following. In the works [13] and [14] the continuation operator is not used explicitly. Instead, an implicit way to prolongate solution from domain $\Omega_{n}$ to the domain $\Omega_{n+1}$ is used. Technically it is implemented by adding to the weak statement of the problem an additional penalty-type bilinear form. As a result, the required smoothness of the discrete extension is a consequence of the modified variational statement of the problem. This method is called "ghost penalty stabilization". It can directly be used to solve the problem considered in this paper. 
Below we use an algorithm, that assumes explicit definition of continuation $\mathrm{E}[u]$ of the solution $u$. Namely, the solution defined in the region $\mathrm{F}_{n}$ is extended to the domain $\mathrm{F}_{n+1}$ as a constant in the direction normal to the boundary of the domain $\mathrm{F}_{n}$.

Alternatively, a continuation approach typical for the X-FEM method can be used (see, for example $[15,16])$. Essentially it consist of two steps:

1. First, the vector field $\mathbf{v}$ defined at the boundary $\partial \mathrm{F}_{t}$ is extended into tubular neighborhood of $\partial \mathrm{F}_{t}$ (or the entire three-dimensional domain containing the surface);

2. Second, the extended velocity field is used to extend solution from the domain $\mathrm{F}_{n}$ into an "extended" domain $\mathrm{F}_{n+1}$ by solving appropriate Hamilton-Jacoby type equation.

At each time step, the problem (6b) is solved on the surface $\mathrm{F}_{n+1}$. A number of methods can be applied to do it, including variational version of the closest point projection method (see [10]).

In what follows, we assume that during its evolution, the surface $\mathrm{F}_{t}$ (and surfaces $\mathrm{F}_{n}$, $n=\overline{0, N}$ ) are always located inside spatial domain $\tilde{\Omega}$. To proceed with spatial approximations, we assume that the finite element mesh $\mathrm{T}_{h}$ is introduced in $\Omega$. This mesh is used to build spatial approximations of the problem (6b) for $n=\overline{1, N}$.

At each moment in time, a three-dimensional domain $\Omega_{n}^{h}$ is associated with the surface $\mathrm{F}_{n}$ in which solution to the problem (6b) will be approximated by the closest point projection method. Geometrically $\Omega_{n}^{h}$ can be constructed as a set of finite elements $\omega \in \mathrm{T}_{h}$, all nodes of which are distant from the surface $\mathrm{F}_{n}$ by a distance not exceeding the value of the given parameter $\delta$, which is a multiple of the step of the computational mesh. Everywhere in the domain $\Omega_{n}^{h}$ we will assume that closest point projector $\mathrm{P}_{n}$ (the index " $n$ " indicates the number of time step) is well defined.

Afterwords to solve the problem in the domain $\Omega_{n+1}$, algorithms from $[17,10,20]$ can be used directly.

The sketch of the computational algorithm is as follows:

1. Initialize surface $F \equiv F_{0}$, velocity field $\mathbf{v}(\mathbf{x})$ defined at $\mathbf{x} \in \partial F$; initial condition $u_{0}(\mathbf{x}), \mathbf{x} \in \mathrm{F}$.

2. Set domain $\Omega$ and the computational mesh $\mathrm{T}_{h}$ in it.

3. Set $n=0, t=0$.

4. Compute discrete approximations of the closest point projector $\mathrm{P}_{0}^{h}$ to the surface $\mathrm{F}_{0}$.

5. For $n=\overline{1, N}$ :

a) Set $n:=n+1$.

b) Define mid-surface boundary evolution velocity $\mathbf{v}$. 
c) Setup mesh domain $\Omega_{n}^{h}$ as a correct subset of the finite elements in $\mathrm{T}_{h}$.

d) Based on the given field $\mathbf{v}$ and the mesh projector $\mathrm{P}_{n-1}^{h}$ determine discrete domain $\Omega_{n}^{h}$ and the corresponding closest point projector $\mathrm{P}_{n}^{h}$. see [18].

e) Compute the extention $\mathrm{E} u_{n-1}$ of the solution from the domain $\Omega_{n-1}^{h}$ to the domain $\Omega_{n}^{h}$.

f) Assemble and solve a finite-dimensional approximation of the problem (6b).

g) Go to step 5a.

6. Terminate algorithm.

Note that during the operation of the algorithm, the surface is specified directly only at its first initialization step. In the further steps, only the evolution over time of the projector $\mathrm{P}_{n}^{h}$ is computed. The image of this projector is an approximation of the surface; Its geometric characteristics, if necessary, can be calculated according to the algorithms described in [18].

To solve discrete non-linear problem at step (5f) of the algorithm, fixed point iterations are used.

As applied to time-implicit approximations of the problem (3) this algorithm, describing transition from the current state $\{p, \rho, w\}$ at the time $t$ to the state $\{\hat{p}, \hat{\rho}, \hat{w}\}$ at the time $t+\Delta t$ has the following form:

1. For $i=0$ set $\hat{p}_{i}=p$.

2. Compute $\hat{w}_{i+1}$ as

$$
\hat{w}_{i+1}(\mathbf{x})=\mathrm{W}\left(\mathbf{x} ; \hat{p}_{i}\right), \quad \hat{\rho}_{i+1}(\mathbf{x})=\rho\left(\hat{p}_{i}(\mathbf{x})\right) .
$$

3. Compute $\hat{p}_{i+1}$ as solution of the equation:

$$
\frac{1}{\Delta t}\left(\rho\left(\hat{p}_{i+1}\right) \hat{w}_{i+1}-\rho w\right)+\operatorname{div}\left(-\frac{1}{12 v} \hat{\rho}_{i+1} \hat{w}_{i+1}^{3} \nabla \hat{p}_{i+1}\right)=f .
$$

4. Check iterations stopping criteria. If the required accuracy is not achieved, set $i:=i+1$ and go to step 2 . If the required accuracy is achieved, set

$$
\{\hat{p}, \hat{\rho}, \hat{w}\}:=\left\{\hat{p}_{i+1}, \rho\left(\hat{p}_{i+1}\right), \mathrm{W}\left(\hat{p}_{i+1}\right)\right\}
$$

It is assumed that the solution is obtained with the required accuracy, if the uniform norm of pressure increment is less then the given threshold value $\varepsilon_{\text {iter }}$ :

$$
\left\|\frac{p_{i}^{n+1}-p_{i-1}^{n+1}}{p_{0}}\right\|_{\infty}<\varepsilon_{\text {iter }} .
$$

\subsection{Spatial approximations with closest point projection method}

To simplify notations let us write down equation (7) as 


$$
\frac{1}{\Delta t} M(u-\tilde{u})+\nabla \cdot(A(\mathbf{x}) \nabla u)=f, \quad \mathbf{x} \in \mathrm{F} .
$$

Following [8] consider continuation of the equation (9) to the spatial domain $\Omega_{\mathrm{F}}$ :

$$
\frac{1}{\Delta t} M(u-\tilde{u})+\nabla \cdot(\mathrm{E}[A](\mathbf{x}) \nabla \mathrm{E}[u])=\mathrm{E}[f], \quad \mathbf{x} \in \mathrm{F} .
$$

The trace of the solution of the embedded equation (10) on $\mathrm{F}$ is the solution of the equation (9).

Boundary conditions for the embedding equation (10) on $\Gamma_{\partial \mathrm{F}}$ (see section 2) can be constructed as an extension of the surface boundary conditions of the original problem (3) defined at $\partial \mathrm{F}$ :

$$
\left.u\right|_{\Gamma_{\partial \mathrm{F}}}=\mathrm{E}\left[\left.u\right|_{\partial \mathrm{F}}\right]=\mathrm{E}[g]
$$

or, which is the same,

$$
u(\mathbf{x})=g(\mathrm{P} \mathbf{x})=g\left(\mathbf{x}_{\mathrm{cp}}\right), \quad \mathbf{x} \in \Gamma_{\partial \mathrm{F}}, \quad \mathbf{x}_{\mathrm{cp}}=\mathrm{P} \mathbf{x} \in \partial \mathrm{F} .
$$

Note that the values of the solution of (10) on $\Gamma_{\mathrm{F}}$ are completely defined as an extension of $u$ from interior points of the surface $\mathrm{F}$. For this reason, the boundary conditions for (10) are not defined on this part of the boundary.

Let us briefly describe now the spatial approximations. Let $\mathrm{T}_{h}=\mathrm{T}_{h}(\Omega)$ be a partition of $\Omega$ into finite elements (tetrahedrons) $\omega$,

$$
\Omega_{h}=\bigcup_{\omega \in \mathrm{T}_{h}} \omega=\Omega,
$$

that is, domain itself and its boundary are approximated exactly. Let $\mathrm{N}$ be a set of triangulation nodes, equipped with simplest continuous piecewise linear basis functions $\varphi_{i}=\varphi_{i}(\mathbf{x}), i \in \mathrm{N}$. The finite-dimensional space $V_{h}(\Omega) \subset V(\Omega)$ can be defined as

$$
V_{h}(\Omega)=\operatorname{span}_{i \in \mathrm{N}} \varphi_{i}(\mathbf{x}) .
$$

Recall that the boundary $\Gamma$ of the domain $\Omega$ is approximated by the computational mesh exactly. Let $\mathrm{N}=\mathrm{N}_{\Omega} \cup \mathrm{N}_{\Gamma}, \mathrm{N}_{\Omega}$ and $\mathrm{N}_{\Gamma}$ be sets of nodes inside domain $\Omega$ and on its boundary $\Gamma$, respectively. Then elements of the space $V_{h}=V_{h}(\Omega)$ have the form

$$
v_{h}(\mathbf{x})=\sum_{i \in \mathrm{N}_{\Omega} \cup \mathrm{N}_{\Gamma}} v_{i} \varphi_{i}(\mathbf{x}) .
$$

To proceed we construct discrete approximation $\mathrm{P}_{h}$ of the closest point projection operator $\mathrm{P}$ first. The details are covered in [18]) and are not considered here. Let $\mathbf{x}_{i}$ be a mesh node in $\Omega_{h}, \mathbf{x}_{i}^{\mathrm{cp}}=\mathrm{P}_{h} \mathbf{x}_{i}$ be its projection, $\omega_{i}$ be a finite element such that $\mathbf{x}_{i}^{\mathrm{cp}} \in \omega_{i}$. Discrete extension operator defines the value of the (extended) function in the node $\mathbf{x}_{i}$ as $u_{h}\left(\mathrm{P}_{h} \mathbf{x}_{i}\right)$. This value 
can be computes using linear interpolation over the values $u_{k}, k \in \omega_{i}$ of $u_{h}$ over nodes $\omega_{i}$. As a result, the value of $u_{h}^{\text {ext }}$ can be defined as

$$
u_{h}^{\mathrm{ext}}=\mathbf{E}_{h} \mathbf{u}_{h}
$$

where $\mathbf{E}_{h}$ is a square matrix which nonzero rows are of the form

$$
\mathbf{E}_{h}\left(i,\left[k_{1}, k_{2}, k_{3}, k_{4}\right]\right)=\left[\xi_{1}\left(\omega_{i}\right), \xi_{2}\left(\omega_{i}\right), \xi_{3}\left(\omega_{i}\right), \xi_{4}\left(\omega_{i}\right)\right] .
$$

Here $\xi_{\alpha}\left(\omega_{i}\right)$ are barycentric coordinates of the point $\mathrm{P}\left(\mathbf{x}_{i}\right)$ inside finite element $\omega_{i}$.

Provided with discrete extension operator, the finite element approximations to (10) (in a case of homogeneous Neumann boundary conditions) reads:

$$
\mathbf{M}_{h} \frac{\hat{\mathbf{u}}_{h}-\mathbf{u}_{h}}{\Delta t}=\mathbf{A}_{h} \mathbf{E}_{h} \mathbf{u}_{h}+\mathbf{E}_{h} \mathbf{f}_{h},
$$

where $\mathbf{M}_{h}$ is the mass matrix, $\mathbf{u}_{h}=\mathbf{u}_{h}(t)$ is a vector of degrees of freedom of the solution at time $t=t_{n} ; \hat{\mathbf{u}}_{h}=\mathbf{u}_{h}(t+\Delta t)$ is vector of degrees of freedom of the solution at time $t=t_{n+1}=t+\Delta t, \mathbf{f}_{h}$ is the right side of the finite-dimensional problem, $\mathbf{A}-$ its stiffness matrix,

$$
\left[\mathbf{M}_{h}\right]_{i j}=\int_{\Omega} M \phi_{i} \phi_{j} d \Omega, \quad\left[\mathbf{A}_{h}\right]_{i j}=\int_{\Omega} \mathrm{E}[A] \phi_{i} \cdot \nabla \phi_{j} d \Omega, \quad\left[\mathbf{f}_{h}\right]_{i}=\int_{\Omega} f \phi_{i} d \Omega, \quad i, j \in \mathrm{N} .
$$

The resulting system of linear algebraic equations has the form:

$$
\left(\mathbf{M}_{h}-\Delta t \mathbf{B}_{h}\right) \hat{\mathbf{u}}_{h}=\Delta t \mathbf{E}_{h} \mathbf{f}_{h}+\mathbf{M}_{h} \mathbf{u}_{h} .
$$

As it is shown in [7], the constructed finite-dimensional problem can be unstable since matrix $\mathbf{B}=\mathbf{A E}$ may have eigenvalues with both positive or negative real part. The same work suggests modification that eliminates this effect. The solution is to modify $\mathbf{B}_{h}$ according to

$$
\tilde{\mathbf{B}}_{h}=\operatorname{diag} \mathbf{A}_{h}+\left(\mathbf{A}_{h}-\operatorname{diag} \mathbf{A}_{h}\right) \mathbf{E}_{h} .
$$

The same modification is used in this work.

A number of approaches can be used to account for boundary conditions in the discrete setting. In this paper, we use the simplest one: the values of the solution in the nodes of the computational mesh, which are projected onto the surface boundary, are assigned according to the given boundary values. Technically, this is done in a standard way, by modification of the appropriate rows of the system of equations above.

The described algorithm is applied at each step of the fixed point iterations to solve the equation (7).

\section{SIMULATION RESULTS}

\subsection{Geometric constructions}

This section provides an illustrations to the basic geometrical constructions needed for application of the closest point projection method (see section 2) using simple example. 
Generally, an algorithmic steps used in closest point projection method are as follows. First, the spatial domain $\Omega$ is defined. The somain contains surface $F$ with boundary $\partial \mathrm{F}$ such that $\overline{\mathrm{F}}=\mathrm{F} \cup \partial \mathrm{F} \subset \Omega$. We consider here the stationary case, i.e., the fracture mid-surface is assumed to be fixed in time and space. Therefore, in this section domains $\Omega$ and $\tilde{\Omega}$ are treated equally.

Hereafter in this work the domain $\Omega$ is asumed to be cube-shaped with an edge of the length $l$. In this domain a tetrahedral mesh is introduced which is constructed as follows: (i) a uniform mesh of $N_{x} \times N_{y} \times N_{z}$ smaller cubes in $\Omega$ is introduced; (ii) each small cube is further divided into five tetrahedra. Those tetrahedrons forms a computational mesh in $\Omega$. In the following it is always assumed $N_{x}=N_{y}=N_{z}=N$. The mesh step size is defined as $h=l / N$. Note that it is not assumed a-priory that the surface $\mathrm{F}$ and the constructed mesh are consistent (i.e., the surface $\mathrm{F}$ is not represented as a union of the mesh faces).

The constructed meshed domain $\Omega^{h}$ is not used in the calculations directly, but is used to build a mesh $\Omega_{\mathrm{F}}^{h}$, in which solutions to the extended (prolongated) equation is to be found.

In accordance with the approach under consideration, the problem (surface PDE) is solved in the meshed domain $\Omega_{\mathrm{F}}^{h}$, which is the "submesh" of the $\Omega^{h}$ so that the distance (in the sense of closest point projection) from all nodes of the mesh in $\Omega_{\mathrm{F}}^{h}$ to the surface does not exceed a given value $\delta$. In all calculations below this value is selected as $\delta=N_{\delta} h$, where $N_{\delta}=4$. Further in this section, the domains $\Omega$ and $\Omega^{h}$ (as well as $\Omega_{\mathrm{F}}^{h}$ and $\Omega_{\mathrm{F}}$ ) are identified and the superscript " $h$ " is dropped, if it does not lead to any misunderstanding.

For the example discussed in this section the surface $\mathrm{F}$ has the form of a flat disk of the diameter $L=1$. The disk is located in the plane $\mathrm{O} x y$ and is centered at the origin. Domain $\Omega$ containing the disk is a cube with edge length $l=1.5$.

On each edge of the cube $\Omega$, a $N=31$ nodes are defined. Thus, the characteristic mesh step size is $h=0.05$.

According to the closest point projection method an equation defined on the surface $F$ is extended and solved in the spatial domain $\Omega_{\mathrm{F}} \subset \Omega$. The region $\Omega_{\mathrm{F}}$ consists of tetrahedrons from $\Omega$ with nodes located at the distance of $\delta=0.2$ or smaller from $\mathrm{F}$. So, the "thickness" of the domain $\Omega_{\mathrm{F}}$ equals to $2 \delta=0.4$ ( $\approx 8$ grid nodes) and its largest diameter is 1.4 ( $\approx 28$ grid nodes).

The nodes of $\Omega_{\mathrm{F}}$ are shown on figure 1 (on the left plot). It also shows a section of the domain $\Omega$ by the plane passing through the center of the disk $F$. The mesh with red edges shows the surface $F$, red spheres denote nodes located in the domain $\Omega_{F}$ ("cloud"). In the same figure (on the right plot) the vectors connecting the mesh nodes $\mathbf{x}$ with their projections $\mathrm{P}(\mathbf{x})$ onto $\mathrm{F}$ are shown. The color of each vector corresponds to its length.

Figure 2 shows the sections of the computational domain by the coordinate planes $\mathrm{O} x y$ and $\mathrm{O} y z$. Red spheres indicate mesh nodes located in $\Omega_{\mathrm{F}}$ which projections belong to the boudary 
$\partial \mathrm{F}$ of the surface. On the same plots the white spheres are nodes in $\Omega_{\mathrm{F}}$, which projections are internal points of the surface $\mathrm{F}$. Blue lines indicate the volumetric mesh defined in $\Omega$.

\subsection{Fluid flow in fracture with fixed mid-surface}

Here we consider the case when the geometry of the fracture does not change in time and fracture opening is pressure dependent. The statement of the problem in this case has the form of (3).

Fracture opening, as a function of pressure, is defined by (4), where reference opening $w_{\text {ref }}(\mathbf{x})$ corresponds to the surface of bi-axial ellipsoid with axes $l_{0}$ and $L_{0}, l_{0}=\iota_{0}$. Here $l_{0}$ is equal to maximum value of fracture opening, see below.

Let $\mathbf{x}_{\mathrm{c}}$ be the center of the fracture, $\mathbf{x}_{\mathrm{cp}}=\mathrm{P}(\mathbf{x})$ - projection of a point $\mathbf{x}$ onto the fracture mid-surface, $d$ is a distance from the center of the fracture to the projection of the point onto the fracture,

$$
d=d(\mathbf{x})=\mathrm{P} \mathbf{x}_{\mathrm{c}}-\mathbf{x}_{\mathrm{cp}} \mathrm{P},
$$

where $\mathrm{P} \cdot \mathrm{P}$ is the Euclidean norm in $\mathrm{R}^{3}$. Then the reference opening as a function of the point at the fracture mid-surface is expressed as

$$
w_{\text {ref }}(\mathbf{x})=l_{0} \sqrt{1-\frac{\mathrm{P} \vec{x}_{\mathrm{c}}-\vec{x}_{\mathrm{cp}} \mathrm{P}^{2}}{L_{0}^{2}}} .
$$

As it was mentioned above, Reynold's lubrication equation (3) is degenerate as opening vanishes at the fracture front (mid-surface boundary). To avoid this situation, we set $L_{0}=(1+\varepsilon) L$ where $L$ is actual radius of the disk-shaped fracture. Here $\varepsilon=l_{0}=L$ is a small parameter.

At the initial state, the pressure is constant over the fracture mid-surface and is equal to $p_{0}$ (reference pressure value). Accordingly, the initial opening distribution equals to the reference one.

Boundary conditions at the fracture front read:

$$
p_{\partial \mathrm{F}}=p_{\partial \mathrm{F}}(\mathbf{x})=p_{0}\left(1+\gamma \frac{x-x_{\mathrm{c}}}{L}\right), \quad \mathbf{x} \in \mathrm{F},
$$

with $\gamma=0.1$ being a parameter, $x$ being projection of the point $\mathbf{x}$ onto the axis $\mathrm{O} x$.

As before, the computational domain is a cube with edge $l=30 \mathrm{~m}$. The mesh step size $h=1 \mathrm{~m}$. The equation is solved in the domain $\Omega_{\mathrm{F}}$ consisting of tetrahedrons, all nodes of which are distant from the fracture mid-surface no further then $\delta=2 \mathrm{~m}$. Time step is $\Delta t=0.5 \cdot 10^{-7} \mathrm{~s}$.

The radius of the fracture is equal to $L=10 \mathrm{~m}$. The fracture is a subset in the plane $\mathrm{O} x y$ and is centered at the origin. The maximum fracture opening $l_{0}=1 \mathrm{~cm}$. 


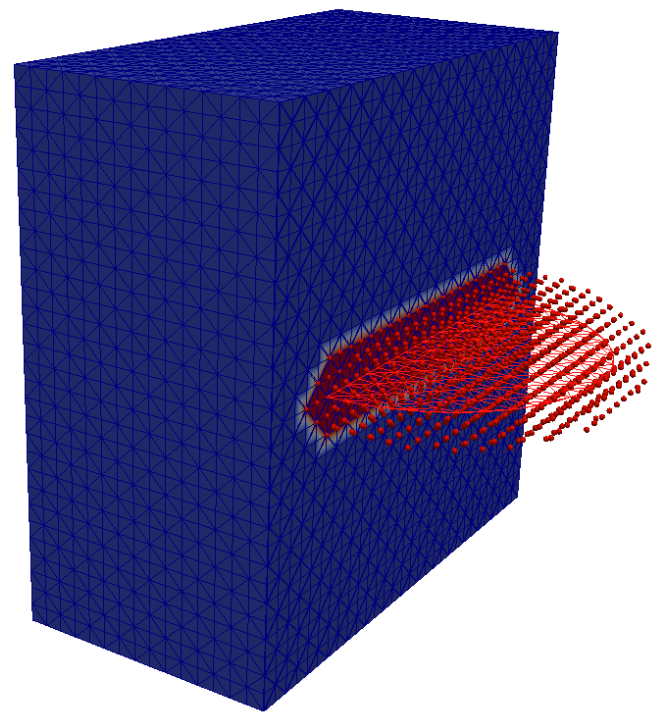

(a)

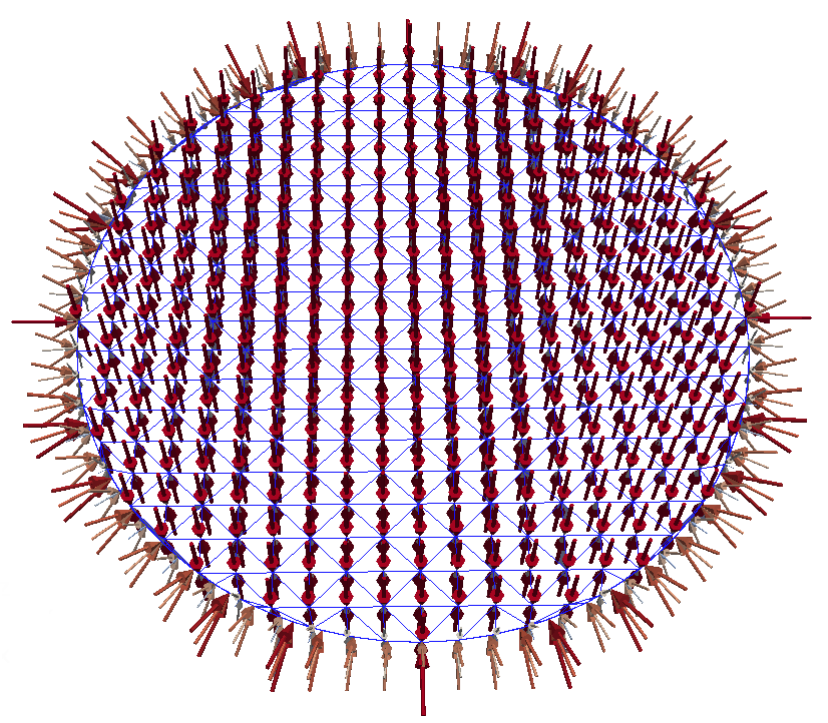

(b)

Figure 1:(a) - section of the domain $\Omega$, surface (red mesh) and mesh nodes in $\Omega_{\mathrm{F}}$ (red markers).

(b) - projection vectors of the nodes inside domain $\Omega_{\mathrm{F}}$ on $\mathrm{F}$.

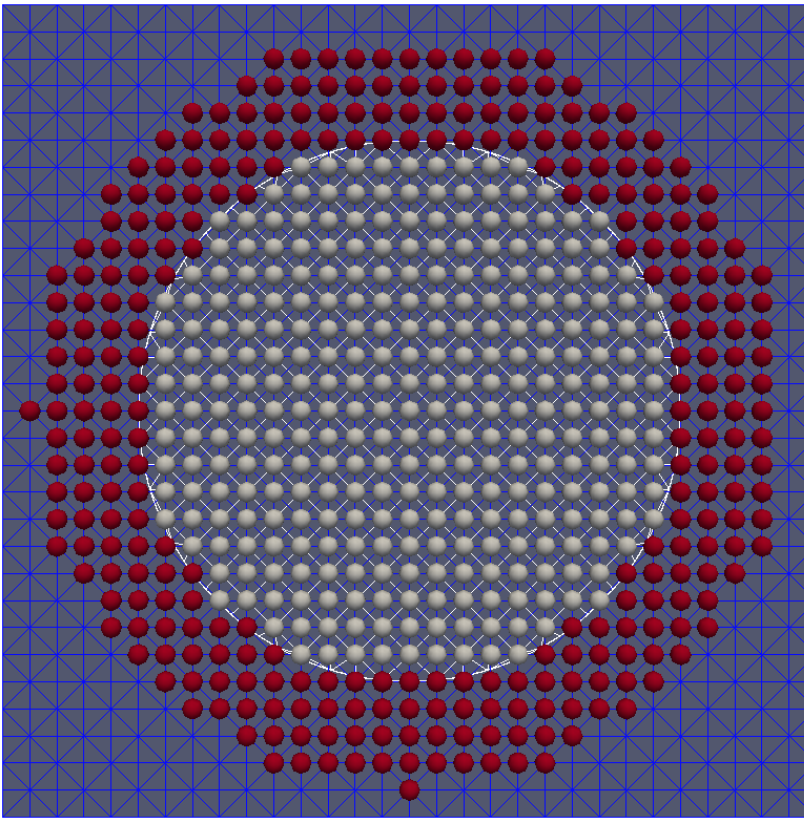

(a)

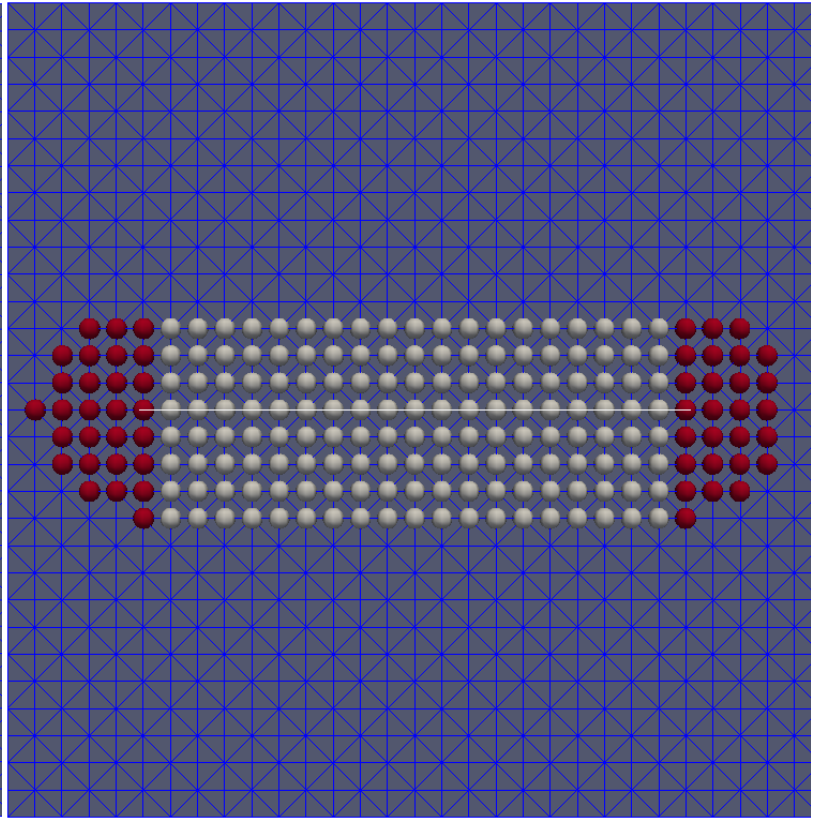

(b)

Figure 2: Projections of the mesh nodes onto the surface (white spheres) and onto its boundary (red speres). The blue lines are edges of computational mesh. (a) - top view, (b) - side view.

In the center of the fracture, inside disk $\Omega_{\mathrm{bc}}^{h}$ of the radius $R=h$ a constant pressure is set being equal to $1.1 p_{0}$. 
Accuracy parameter in (8) is set to $\varepsilon_{\text {iter }}=1.0 \cdot 10^{-6}$ and the maximum number of iterations is $N_{\text {iter }}^{\max }=10$.

Physical parameters of the fluid in fracture are as follows: kinematic viscosity $v=1 \cdot 10^{-3} \mathrm{~Pa} \cdot \mathrm{s}$, reference pressure $p_{0}=300$ bar, reference opening $l_{0}=1 \mathrm{~cm}$, compressibility $c_{\mathrm{f}}=4.16 \cdot 10^{-10} \mathrm{~Pa}^{-1}$, density $\rho_{0}=1000 \mathrm{~kg} / \mathrm{m}^{3}$. Parameters of the equation (4) are $c_{\mathrm{w}}=1 / p_{0}, \quad p_{\text {ref }}=p_{0}$.

Pressure distribution and fracture opening for the computed solution for different moments of time are shown on figure 3 .

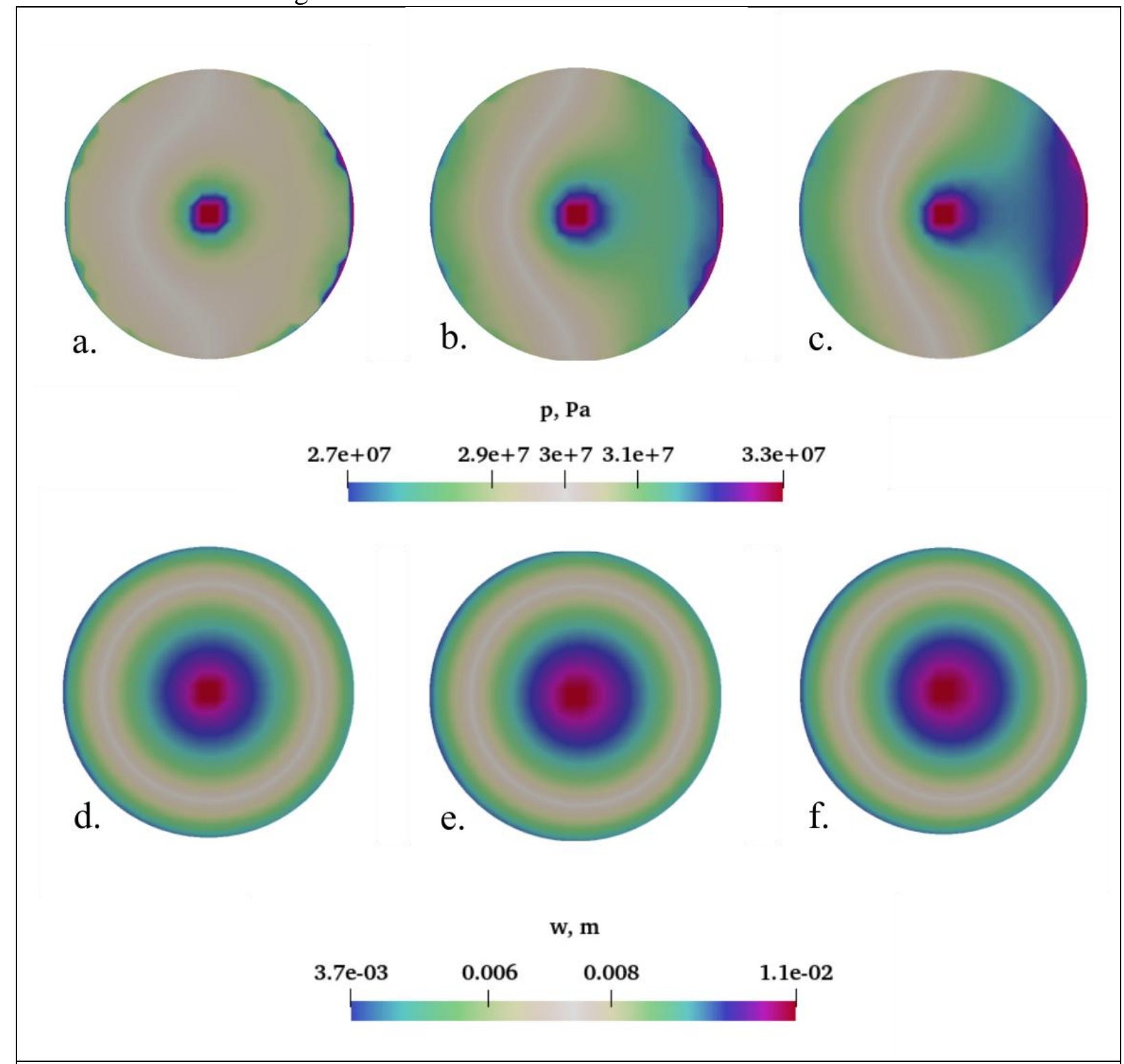

Figure 3: Pressure (on the top) and fracture opening (on the bottom) distribution at time $t=\Delta t$ (a, d), $t=3 \Delta t(\mathrm{~b}, \mathrm{e}), t=5 \Delta t(\mathrm{c}, \mathrm{f})$. 


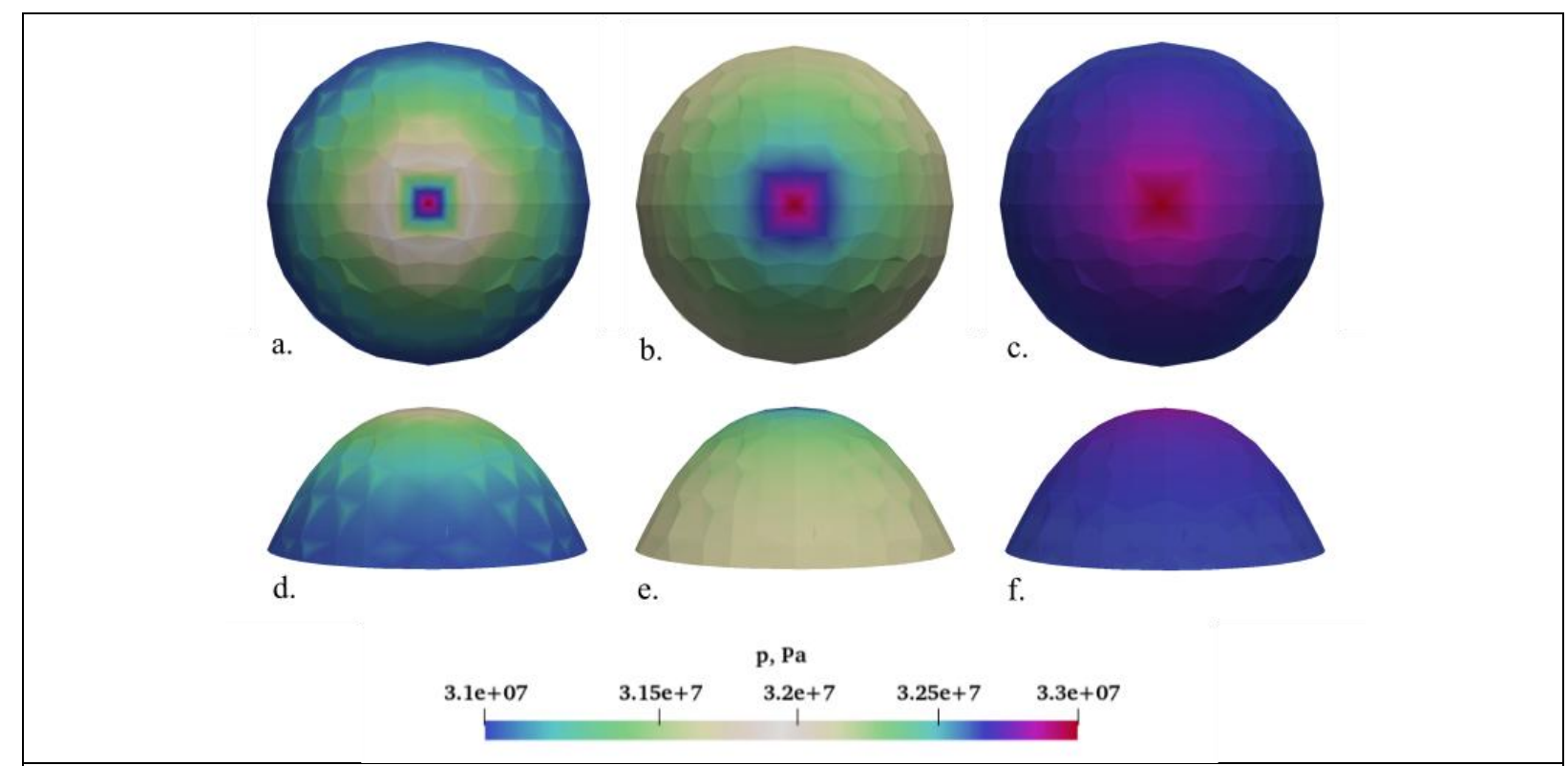

Figure 4: Pressure distribution at time $t=\Delta t(\mathrm{a}, \mathrm{d}), t=3 \Delta t(\mathrm{~b}, \mathrm{e}), t=5 \Delta t(\mathrm{c}, \mathrm{f})$. Top: top view, bottom: side view.

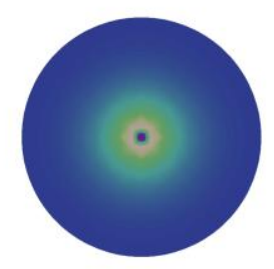

a.

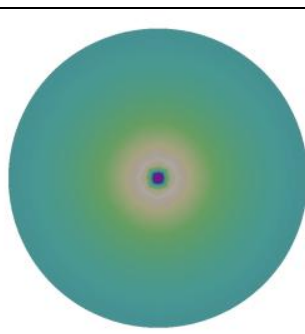

b.

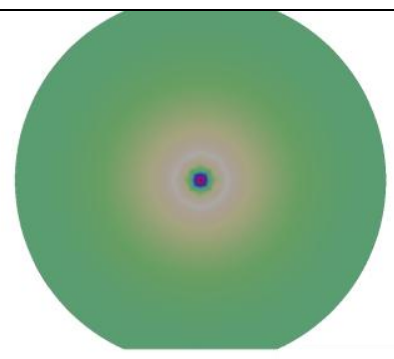

c.

p, Pa

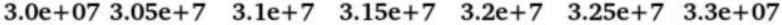

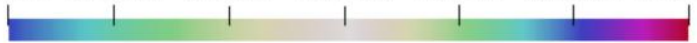

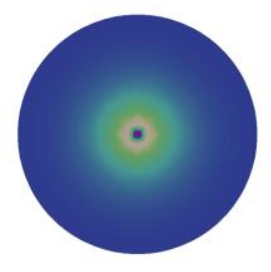

d.

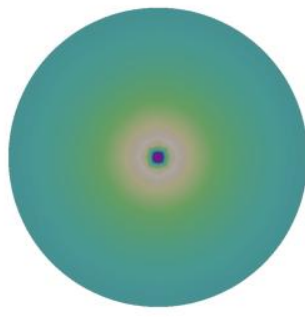

e.

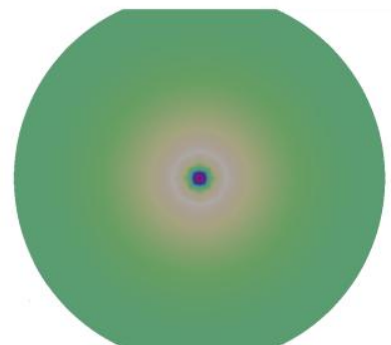

f.

w, m

$\begin{array}{llllll}1.0 \mathrm{e}-02 & 0.0102 & 0.0104 & 0.0106 & 0.0108 & 1.1 \mathrm{e}-02\end{array}$

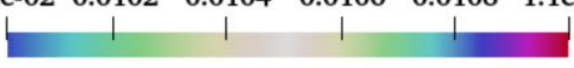

Figure 5: Pressure (top) and fracture opening (bottom) distribution at time $t=\Delta t(\mathrm{a}, \mathrm{d}), t=6 \Delta t(\mathrm{~b}, \mathrm{e}), t=$ $12 \Delta t(\mathrm{c}, \mathrm{f})$. 
The presented simulation results show that to achive the required accuracy using fixed-point iteration method requires no more than 10 iterations for each time layer. As expected, the number of iterations significantly decreased with time.

Next, we consider the case of fracture with fixed geometry but of the complex shape. The statement of the problem is the same in general.

At the initial moment of time, the pressure is constant over the middle surface of the fracture and is equal to $p_{0}$ (reference pressure value). The opening is constant as a function of the mid-surface point and is equal to $l_{0}$ (reference value of the opening). Dependency of the opening on the pressure is given by (4) with $w_{\text {ref }}(\mathbf{x})=l_{0}=$ const .

The computational domain is a cube with edge length $l=50 \mathrm{~m}$, mesh step size is $h=1 \mathrm{~m}$. The equation is solved in the domain $\Omega_{\mathrm{F}}$, consisting of tetrahedrons, all nodes of which are distant from the fracture no further than $\delta=2 \mathrm{~m}$. Time step size is $\Delta t=0.00024 \mathrm{~s}$.

Accuracy parameter in fixed point iterations stopping criteria is equal to $\varepsilon_{\text {iter }}=1.0 \cdot 10^{-6}$, the maximum number of iterations is set to $N_{\text {iter }}^{\max }=3$.

Physical parameters of the fluid are as followos: kinematic viscosity $v=1 \cdot 10^{-3} \mathrm{~Pa} \cdot \mathrm{s}$, reference pressure value $p_{0}=300$ bar, reference opening $l_{0}=1 \mathrm{~cm}$, compressibility $c_{\mathrm{f}}=1 \cdot 10^{-6} \mathrm{~Pa}^{-1}$, density $\rho_{0}=1000 \mathrm{~kg} / \mathrm{m}^{3}$. Parameters in equation (4) are $c_{\mathrm{w}}=1 / p_{0}$, $p_{\text {ref }}=p_{0}$.

The fracture mid-surface is part of the paraboloid of the height $3 \mathrm{~m}$ and radius of lower section $4 \mathrm{~m}$. At the top of the paraboloid, in the disk $\Omega_{\mathrm{bc}}^{h}$ of the radius $R=h$, where $h$ is characteristic grid step, constant pressure equal to $1.1 p_{0}$ is set over time. The simulation results are presented on figure 4.

\subsection{Fluid flow in evolving fracture}

In this section a case of fluid flow in evolving fracture is considered.

We assume that fracture opening depends on pressure in accordance with the equation (4).

Evolution of the fracture is driven by axially symmetric velocity field $\mathbf{v}$ given by

$$
\mathbf{v}(\mathbf{x}, t)=v_{\mathrm{m}} \frac{\mathbf{x}-\mathbf{x}_{\mathrm{m}}}{\mathrm{P} \mathbf{x}-\mathbf{x}_{\mathrm{m}} \mathrm{P}}, \quad \mathbf{x} \in \partial \mathrm{F}_{t} .
$$

As it can be seen, fracture evolution occurs in the plane $\mathrm{O} x y$. The velocity field is defined by the point of the "growth center" $\mathbf{x}_{\mathrm{m}}$ and a constant (i.e., independent of the moment of time and point of space) velocity $v_{\mathrm{m}}$. For time interval $\Delta t$ the point $\mathbf{x}$ of the fracture front moves according to

$$
\mathbf{x}(t+\Delta t)=\mathbf{x}(t)+\Delta t v_{\mathrm{m}} \frac{\mathbf{x}(t)-\mathbf{x}_{\mathrm{m}}}{\left\|\mathbf{x}(t)-\mathbf{x} r_{\mathrm{m}}\right\|}
$$


It is assumed that domain $\Omega$ is sufficiently large and contains fracture mid-surface at each moment of time during fracture evolution. Domain $\Omega_{\mathrm{F}}$, where the problem is actually solved, evolves according to fracture evolution.

The computational domain is a cube with edge $l=50 \mathrm{~m}$. Accordingly, the mesh step size is $h=1 \mathrm{~m}$. The equation is solved in the domain $\Omega_{h}^{n}$, consisting of tetrahedrons, all nodes of which are distant from the fracture not further than $\delta=2 \mathrm{~m}$. Time step is set to $\Delta t=0.5 \cdot 10^{-7}$ s.

The radius of the fracture at the initial moment of time $L=10 \mathrm{~m}$, the fracture belongs to plane $\mathrm{O} x y$ and is centered at the origin. The fracture growth velocity $v_{\mathrm{m}}$ is chosen so that $v_{\mathrm{m}} \Delta t=0.5 \mathrm{~m}$. The reference opening is assumed to be constant over fracture surface, $w_{\text {ref }}(\mathbf{x})=$ const .

The physical parameters are as follows: kinematic viscosity of the fluid $v=1004 \cdot 10^{-6} \mathrm{~Pa} \cdot \mathrm{s}$, reference pressure value $p_{0}=300$ bar, fluid compressibility $c_{\mathrm{f}}=4.16 \cdot 10^{-10} \mathrm{~Pa}^{-1}$, density $\rho_{0}=1000 \mathrm{~kg} / \mathrm{m}^{3}$. The reference value of the opening is $w_{\text {ref }}=10 \mathrm{~mm}$. Parameters of the equation (4) are $c_{\mathrm{w}}=1 / p_{0}, p_{\text {ref }}=p_{0}$.

In the center of the fracture, in the disk $\Omega_{\mathrm{bc}}$ of the radius $R=h$, with $h$ being the characteristic mesh step size, a constant in time pressure equal to $1.1 p_{0}$ is defined. At the initial time, the pressure in the fracture is equal $p_{0}$.

Accuracy parameter in fixed point iterations stopping criteria is equal to $\varepsilon_{\text {iter }}=1.0 \cdot 10^{-6}$. The maximum number of iterations is $N_{\text {iter }}^{\max }=10$.

The simulation results are presented in the figure 5.

\section{CONCLUSION}

The present work is devoted to the numerical study of the finite element version of the closest point projection method applied to the numerical solution of the Reynol'd lubrication equations which describes fluid flow in fractures. Both stationary and evolving fractures are considered. The key idea of the approach is to apply closest point projection method to describe surface evolution and to construct embedding PDE. The fracture mid-surface evolution is described entirely in terms of discrete closest point projection operator without use of any other representation of the fracture. A number of test problems are considered, for stationary and evolving fractures. Numerical experiments demonstrated has demonstrated robustness and efficiency of the overall approach.

\section{REFERENCES}

[1] Economides Michael J., Oligney Ronald E., Valko P. Unified Fracture Design: Bridging the Gap Between Theory and Practice, Orsa Press, 2001.

[2] Savenkov E.B., Borisov V.E., "A mathematical model for hydraulic fracture propagation in three dimensional poroelastic medium", PNRPU Mechanics Bulletin, 1, 5-17 (2018),

[3] Poveschenko Y.A., Podryga, V.O., Rahimly, P.I, "About one approach to free-volumetric approximation of a piezoconductive medium with gas hydrate inclusions", Mathematica Montisnigri, 40, 68-89 (2017). 
[4] Ruuth, S.J., Merriman, B., "A simple embedding method for solving partial differential equations on surfaces", J. Comput. Phys., 227, 1943-1961 (2008).

[5] Merriman, B., Ruuth, S.J., "Diffusion generated motion of curves on surfaces", J. Comput. Phys., 225, 2267-2282 (2007).

[6] Macdonald, C.B., Ruuth, S.J., "Level set equations on surfaces via the Closest Point Method", $J$. Sci. Comput., 35, 219-240 (2008).

[7] Macdonald, C.B., Ruuth, S.J., "The implicit Closest Point Method for the numerical solution of partial differential equations on surfaces", SIAM J. Sci. Comput., 31, 4330-4350 (2009).

[8] Macdonald, C.B., Brandman, J., Ruuth, S.J., "Solving eigenvalue problems on curved surfaces using the Closest Point Method", J. Comput. Phys., 230, 7944-7956 (2011).

[9] März, T., Macdonald, C.B., "Calculus on Surfaces with General Closest Point Functions", SIAM J. Numer. Anal., 50(6), 3303-3328 (2012).

[10] Savenkov, E. B., "Finite element framework for closest point projection method for surface PDEs", Keldysh Institute preprints. 8 (2020).

[11] Ramazanov, M. M.,Kritskii, B. V., Savenkov, E. B., "Formulation of the j-integral for the biot elastic porous medium model", J Eng Phys Thermophys, 91 (2018).

[12] Ramazanov, M., Borisov, V., Kritsky, B., Savenkov, E., "Fracture growth criterion for poroelastic media", AIP Conference Proceedings, 2051 (2018).

[13] Lehrenfeld, C., Olshanskii, M., "An Eulerian finite element method for pdes in time-dependent domains", ESAIM: M2AN, 53(2):585-614 (2019).

[14] Burman, E., Frei, S., Massing, A., "Eulerian time-stepping schemes for the non-stationary Stokes equations on time-dependent domains", arXiv preprint, arXiv:1910.03054 (2019).

[15] Moës, N., Gravouil, A., Belytschko, T., "Non-planar 3D crack growth by the extended finite element and level sets - Part I: Mechanical Model", Int. J. Num. Meth. Eng., 53(11), 2549-2568 (2002).

[16] Gravouil, A., Moës, N., Belytschko, T., "Non-planar 3D crack growth by the extended finite element and level sets - Part II: Level set update", Int. J. Num. Meth. Eng., 53(11), 2569-2586 (2002).

[17] Savenkov, E. B., "Numerical techniques for surface PDEs. Review of approaches", Keldysh Institute preprints, 5 (2020).

[18] Ivanov, A.V., Savenkov, E.B., "Simulation and visualization of the dynamics of a surface with a movable boundary on a stationary unstructured mesh", SciVis, 9(2), 64-81 (2017).

[19] Zipunova, E. V., Savenkov E. B., "Solving Reynolds lubrication equations using closest point projection method", Keldysh Institute preprints, 10 (2020).

[20] Zipunova, E.V., Ivanov, A.V., Savenkov, E.B., "Solution of Reynolds lubrication equation on evolving surfaces", Keldysh Institute preprints, 13 (2020).

[21] B.A. Dubrovin, A.T. Fomenko, S.P. Novikov, Modern Geometry - Methods and Applications, Springer, Vol.104, (1984-1990).

Received January 12, 2020 